\title{
LESIONES MÚSCULO ESQUELÉTICAS CATASTRÓFICAS EN CABALLOS PURA SANGRE DE CARRERA, CARACAS-VENEZUELA
}

\author{
Catastrophic Musculoskeletal Injuries in Thoroughbred Horses, \\ Caracas-Venezuela
}

\begin{abstract}
Abelardo Morales B. ${ }^{1,2}$, Víctor Bermúdez G. ${ }^{1}$, Francisco García G. ${ }^{1}$, Miguel Perdigón M. $^{3}$ y Raúl Naranjo B. ${ }^{3}$
\end{abstract}

\section{ReSUMEN}

\begin{abstract}
Se hizo un estudio clínico-patológico de las lesiones músculo-esqueléticas catastróficas ocurridas en pruebas públicas en el Hipódromo "La Rinconada", Caracas, Venezuela, entre el 2000 al 2007. Se trabajó con 78 equinos Pura Sangre de Carrera con lesiones del aparato locomotor. Se colectó muestras de sangre para estudio hematológico, bioquímico y hormonal (paratohormona), así como muestras de orina para determinar niveles de fosfatos. Se les practicó la eutanasia debido a la severidad de las lesiones y se realizó la necropsia. Se tomó secciones de tejido óseo para determinación de calcio y fósforo, y de tejido muscular, tendinoso, gástrico, renal y hepático para estudio histológico. Los exámenes clínicos revelaron claudicación grado IV-V en todos los animales. La pérdida de continuidad ósea (fracturas) ocurrió en todos los casos, y exposición de estructuras óseas, articulares, rupturas de tendones, ligamentos y compromiso vascular en el $60 \%$. Las fracturas fueron de tipo multifragmentaria $(60 \%)$, oblicua $(25 \%)$ y espiral $(15 \%)$. Los valores sanguíneos y de orina estuvieron afectados. En la necropsia se observó el síndrome ulceroso gástrico equino, congestión hepática y necrosis tubular aguda renal. El análisis histológico reveló gastritis aguda superficial severa, úlceras gástricas, necrosis periacinar hepática, osteomalacia y necrosis del hueso subcondral, condromalacia y capsulitis severa.
\end{abstract}

Palabras clave: fractura, lesiones óseas, equino

\section{ABSTRACT}

A clinical and pathological study was conducted on musculoskeletal catastrophic lesions of the locomotive apparatus occurred in 78 Thoroughbred horses in the racetrack «La Rinconada», Caracas, Venezuela, during 2000 to 2007. Blood samples were collected for hematological, biochemical and parathormone analysis in blood. Also, urine samples for phosphate levels. All animals were euthanized due to severe lesions, and bone,

\footnotetext{
${ }^{1}$ Departamento de Patología Veterinaria, Facultad de Ciencias Veterinarias, Universidad Central de Venezuela-UCV. Venezuela

${ }^{2}$ E-mail: aamorales13@gmail.com

${ }^{3}$ Departamento de Patología y División de Inspección Veterinaria, Instituto Nacional de Hipódromos "La Rinconada", Caracas, Venezuela
} 
tendons, stomach, kidney and liver samples were taken for by histopathology evaluation. Clinical exams revealed lameness grade IV-V in all animals. Losses of bone continuity (fracture) occurred in all cases, and exposition of bone structures, joint damage, rupture of tendons and ligaments and vascular destruction were observed in $60 \%$ of them. The types of fractures were comminuted (60\%), oblique (25\%) and spiral (15\%). Blood and urine values were affected. Necropsy showed equine gastric ulcer syndrome, liver congestion and renal acute tubular necrosis. Histopathological analysis showed acute and severe gastritis, gastric ulcers, hepatic periacinar necrosis, osteomalacia and necrosis in subchondral bone, chondromalacia and severe capsulitis.

Key words: fracture, bone lesions, equine

\section{INTRODUCCIÓN}

Las lesiones del aparato locomotor en el caballo de carrera corresponden a la casuística clínica más común en este tipo de animales (Peloso et al., 1994). La exigencia y demanda física durante el ejercicio y la carrera compromete el tejido óseo, articular, muscular y tendinoso en la biomecánica de movimiento (Nixon, 1996). Lesiones músculo-esqueléticas resultantes de las carreras (Peloso et al., 1994) constituyen una causa común de bajo rendimiento y la mayor causa de fatalidad en carreras en Inglaterra (Williams et al., 2001). Estas lesiones resultan, en la mayoría de casos, en eutanasia debido al compromiso vascular (Estberg et al., 1998).

Las lesiones músculo-esqueléticas obedecen a causas multifactoriales, entre ellas la conformación, edad, nutrición, distancia e intensidad de entrenamiento, y condiciones de la pista. La edad del inicio de la actividad física coincide con la etapa final del desarrollo y crecimiento músculo esquelético. La conformación es, en la actualidad, un indicador importante de la capacidad atlética, existiendo características especificas de conformación que se evalúan tanto de manera subjetiva como objetiva (mediante la medición de puntos y ángulos anatómicos) (Anderson et al., 2004). Asimismo, las alteraciones de conformación anatómica son corregibles al nacimiento mediante técnicas quirúrgicas y arreglos podales (Back et al., 2001).

El entrenamiento progresivo permite la capacidad adaptativa músculo esquelética, especialmente para el desarrollo de fibras musculares tipo I y II-B (Sucre et al., 1999). Los caballos con mayor desarrollo de fibras I (contracción lenta), tienen mayor capacidad para correr distancias superiores a los 1400 - $1800 \mathrm{~m}$, en tanto que aquellos con mayor desarrollo de fibras II-A y II-B (fibras de contracción rápida) se desempeñan mejor en carreras cortas (400-1200 m) (Sucre et al., 1999). La intensidad del ejercicio predispone la ocurrencia de microfracturas, desbalance de las fuerzas de carga, estrés traumático y fracturas que pueden ser fatales (Nixon, 1996). Asimismo, es común la presencia de hiperparatiroidismo secundario asociado a enfermedad renal crónica y al uso prolongado de AINES (antinflamatorios no esteroideos) como fenilbutazona, flunixin meglumine y glucocorticoides (dexametasona) y diuréticos (furosemidas), solos o en conjunto con otros fármacos en caballos de alta competencia.

En consideración a estos problemas, se planteó el presente estudio para hacer una descripción clínico-patológica de las lesiones músculo-esqueléticas catastróficas durante pruebas públicas en el Hipódromo "La Rinconada" en el periodo 2000-2007. 
Cuadro 1. Ocurrencia de fracturas en caballos Pura Sangre de Carrera en el Instituto Nacional de Hipódromos "La Rinconada", Caracas-Venezuela, según sexo, edad, y exposición de tejido óseo, blandos y compromiso vascular

\begin{tabular}{|c|c|c|c|c|c|c|c|}
\hline \multirow[b]{2}{*}{ Año } & \multirow{2}{*}{$\begin{array}{l}\mathrm{N}^{\mathrm{o}} \mathrm{de} \\
\text { fracturas }\end{array}$} & \multicolumn{2}{|c|}{ Sexo } & \multicolumn{2}{|c|}{ Edad (años) } & \multicolumn{2}{|c|}{ Tipo de fracturas } \\
\hline & & Macho & Hembra & $\leq 3$ & $>3$ & $\begin{array}{c}\text { No } \\
\text { expuesta }\end{array}$ & Expuesta \\
\hline 2000 & 3 & 1 & 2 & 0 & 3 & 1 & 2 \\
\hline 2001 & 2 & 0 & 2 & 1 & 1 & 0 & 2 \\
\hline 2002 & 10 & 3 & 7 & 4 & 6 & 5 & 5 \\
\hline 2003 & 14 & 4 & 10 & 5 & 9 & 4 & 10 \\
\hline 2004 & 22 & 8 & 14 & 10 & 12 & 8 & 14 \\
\hline 2005 & 10 & 4 & 6 & 5 & 5 & 4 & 6 \\
\hline 2006 & 11 & 4 & 7 & 4 & 7 & 6 & 5 \\
\hline 2007 & 6 & 3 & 3 & 2 & 4 & 3 & 3 \\
\hline Total & 78 & 27 & 51 & 31 & 47 & 31 & 47 \\
\hline
\end{tabular}

\section{Materiales y Métodos}

Se realizó un estudio clínico-patológico a 78 ejemplares Pura Sangre de Carrera (PSC) (27 machos y 51 hembras), con edades comprendidas entre 2 y 6 años, que presentaron lesiones del aparato locomotor, de pronóstico reservado, en pruebas públicas en el Instituto Nacional de Hipódromos (INH) "La Rinconada", Caracas-Venezuela, entre los año 2000 y 2007.

Se colectó muestras de sangre a todos los ejemplares para la determinación de proteínas plasmáticas (modificación de la reacción de Biuret) y hemoglobina (cianmetahemoglobina), así como creatinina, BUN, creatinacinasa y fosfatasa alcalina mediante métodos convencionales de laboratorio clínico. Se midieron los niveles de la paratohormona (PTH), minerales séricos (calcio y fósforo) y se tomaron muestras de orina para determinar fosfato urinario. Asimismo, se tomaron placas radiográficas en 50 casos.
Todos los ejemplares fueron sacrificados (Melling y Alder, 1998) y la necropsia se realizó en el Departamento de Patología Veterinaria del INH "La Rinconada". Se colectó nuestras de tejido óseo, muscular, tendinoso, gástrico, renal y hepático para el estudio histopatológico, y fueron procesados por métodos convencionales. Asimismo, se tomaron secciones de tejido óseo paras análisis de calcio y fósforo.

\section{Resultados}

Los exámenes clínicos revelaron claudicación grado IV-V en todos los casos estudiados. Las lesiones ocurrieron con mayor frecuencia en los miembros anteriores (69/ 78, Fig. 1). El miembro anterior izquierdo fue el más afectado $(61.5 \%, 48 / 78)$, seguido del miembro anterior derecho $(26.9 \%, 21 / 78)$, miembro posterior izquierdo $(9.0 \%, 7 / 78) \mathrm{y}$ miembro posterior izquierdo $(1.3 \%, 1 / 78)$. 


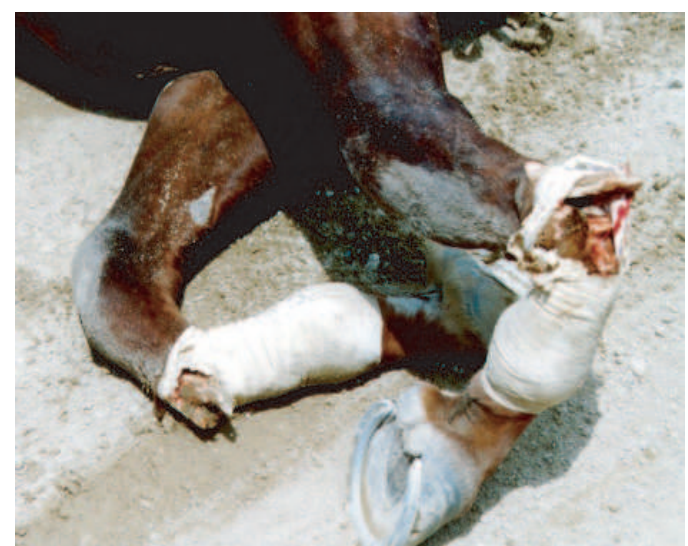

(a)

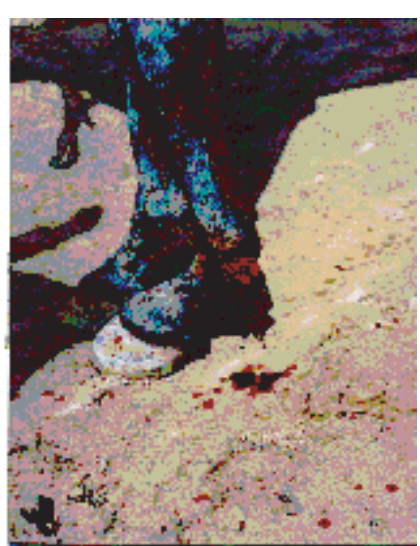

(b)

Figura 1. (a) Pérdida de la continuidad ósea, multifragmentaria, y ruptura de ligamentos con compromiso vascular del metacarpo principal miembro anterior izquierdo y derecho. (b) Pérdida de la continuidad ósea y ruptura de ligamentos con compromiso vascular de la articulación metacarpo-falangiana del miembro anterior izquierdo.

Las bases anatómicas más afectadas fueron los carpos y la articulación metacarpofalangiana (88.5\%, 69/78, Fig. 1b), seguido de fracturas de huesos sesamoideos proximales $(55.1 \%, 43 / 78)$, fracturas del metacarpo $(16.7 \%, 13 / 78)$ y fracturas de huesos del carpo $(11.5 \%, 9 / 78)$. La pérdida de continuidad ósea se presentó en los 78 casos. La fractura completa (abierta) con exposición de estructuras óseas, articulares, rupturas de tendones, ligamentos y compromiso vascular del miembro afectado ocurrió en 47 casos (Cuadro 1). El tipo de fractura, según la descripción morfológica fue multifragmentarias o conminutas $(60.2 \%, 47 /$ $78)$, oblicuas o en pico de flauta $(32.0 \%, 25 /$ $78)$ y espiral $(17.9 \%, 14 / 78)$. El mayor número de fracturas se presentó en hembras y en animales de mayor edad ( $>3$ años) (Cuadro 1).

Los estudios radiológicos evidenciaron microfracturas y fracturas óseas. Además, se observó osteofitos, microfracturas y remodelación laminar ósea en 10 de las 50 radiografías a nivel del metacarpo principal, características radiológicas consistentes con enfermedad periosteal del tercer metacarpiano. Además, se observó lesiones asociadas a enfermedad degenerativa articular
Cuadro 2. Valores hematológicos, bioquímicos $\mathrm{y}$ contenido mineral en muestras de sangre de caballos Pura Sangre de Carrera con fracturas en miembros

\begin{tabular}{ll}
\hline Variable & Valor \\
\hline $\begin{array}{l}\text { Proteínas } \\
\text { plasmáticas }\end{array}$ & $5-9 \mathrm{~g} / \mathrm{dl}$ \\
Hemoglobina & $16 \mathrm{~g} / \mathrm{dl}$ \\
Creatinina & $2-5 \mathrm{mg} / \mathrm{dl}$ \\
BUN & $40-50 \mathrm{mg} / \mathrm{dl}$ \\
CK & $500-650 \mathrm{UI} / 1$ \\
Fosfatasa alcalina & $110-180 \mathrm{UI} / 1$ \\
Calcio & $10-12 \mathrm{mg} / \mathrm{dl}$ \\
Fósforo & $5-8 \mathrm{mg} / \mathrm{dl}$ \\
Ca / P & Ca: $5-12 \mathrm{mg} / \mathrm{dl}$ \\
Ca óseo & $\mathrm{P}: 9 / 12.6 \mathrm{mg} / \mathrm{dl}$ \\
P óseo & $142.8 \pm 2 . \mathrm{mg} / \mathrm{ml}$ \\
Paratohormona & $262.7 \pm 5.2 \mathrm{mg} / \mathrm{ml}$ \\
\hline
\end{tabular}

$(40 \%, 20 / 50)$ (disminución del espacio articular, osteofitos y quistes óseos subcondrales). 
Los resultados del análisis de sangre se muestran en el Cuadro 2. Una ligera alteración de calcio óseo $(110.5 \pm 1.4 \mathrm{mg} / \mathrm{ml})$ y de fósforo $(390.5 \pm 3.4 \mathrm{mg} / \mathrm{ml})$ se observó en 28 animales (36\%). Los niveles de excreción urinaria de fósforo fueron mayores a $30 \mathrm{mg} / \mathrm{dl}$.

Todos los cortes histológicos de tejido óseo mostraron focos de condromalacia, osteomalacia, osteoartritis y capsulitis. El hígado presentó necrosis periacinar e hiperplasia ductal biliar $(57.7 \%, 45 / 78)$. En el tejido renal se encontró nefritis intersticial $(44.9 \%, 35 / 78)$ y glomerulonefritis membranoproliferativa $(32.0 \%, 25 / 78)$. Además, se observó gastritis aguda superficial $(44.9 \%, 35 / 78)$ y úlceras gástricas $(55.1 \%$, 43/78). Dos animales evidenciaron úlceras a nivel del colon.

\section{Discusión}

Las lesiones del aparato locomotor en el equino son una de las principales causas de retiro temporal o total de las competencias, e inclusive de la eutanasia. Esto afecta seriamente la productividad de la industria equina.

La presentación de lesiones por año fue variable, y pueden deberse a las condiciones de la pista y los efectos de año con mayor periodo de invierno y lluvias. La mayor frecuencia de fracturas en miembros anteriores y de los huesos del carpo a distal encontradas en el presente estudio coincide con lo señalado por Rose y Hodgson (1995). Asimismo, la relación entre fracturas de miembros anteriores/posteriores y derechos/izquierdos concuerda con otros estudios (Peloso et al., 1994; Rose y Hodgson, 1995; Williams et al., 2001; Riggs, 2002; Boden et al., 2006). El mayor número de casos en hembras fue producto de una mayor proporción de ellas en las carreras en estudio.

La alteración de los niveles de calcio y fósforo óseo puede predisponer la ocurrencia de fracturas, y se pudo observar en el
$36 \%$ de los animales con fracturas. La elevada excreción urinaria de fósforo $(>30 \mathrm{mg} / \mathrm{dl})$ indica niveles altos de este mineral a nivel del pasto, concentrado o en los suplementos. Esto altera la relación calcio/fósforo pudiendo conllevar a cuadros de hiperparatiroidismo secundario nutricional y remodelación ósea, afectando las concentraciones de minerales ( $\mathrm{Ca}, \mathrm{P}, \mathrm{Mg}, \mathrm{Si}$, entre otros) en el hueso, favoreciendo las fracturas por estrés o fatiga del tejido. Adicionalmente, el uso de diuréticos de asa, AINES y corticoides puede complicar el desbalance metabólico, considerando que se observó daño hepático en los cortes histológicos, consistentes con toxicidad por AINES y daño renal indicativo de enfermedad renal crónica. Esto es un factor determinante del debilitamiento óseo (desmineralización) por alteración en la reabsorción de calcio, fósforo y otros minerales y del desarrollo de fracturas por estrés. Los niveles alterados de la bioquímica sanguínea están posiblemente asociados a la lesión presentada y al estrés del ejercicio por la liberación de catecolaminas, ya que los ejemplares se encontraban en competencia.

Lesiones gástricas se observaron en todos los equinos, con mayor prevalencia de úlceras $(55.1 \%)$ y gastritis $(44.9 \%)$. A nivel del colon ha sido reportada una alta prevalencia de lesiones ulcerativas (Rose y Hodgson, 1995), aunque la frecuencia de estas lesiones fueron bajas en el presente estudio. La mayor ocurrencia de lesiones en caballos de mayor edad se podría atribuir a patologías músculo-esqueléticas crónicas como tendinitis crónica, enfermedad degenerativa articular y microfracturas de cartílago, algunas de las cuales se observaron en la evaluación radiológica. Esto predispone a lesiones severas y fracturas por estrés o fatiga.

\section{Agradecimientos}

Los autores expresan su agradecimiento al Consejo de Desarrollo Científico y Humanístico de la Universidad Central de Venezuela y a la Dirección de Desarrollo y Fomento Equino del Instituto Nacional de Hipódromos "La Rinconada" Caracas, Venezuela. 


\section{Literatura Citada}

1. Anderson T, Mcllwraith CW, Douay P. 2004. The role of conformation in musculoskeletal problems in the racing Thoroughbred. Equine Vet J 36: 571-575.

2. Back W, Clinton, H. 2001. Equine locomotion. London: WB Saunders. $400 \mathrm{p}$.

3. Boden LA, Anderson GA, Charles JA, Morgan KL, Morton JM, Parkin TD, Slocombe RF, Clarke AF. 2006. Risk of fatality and causes of death of Thoroughbred horses associated with racing in Victoria, Australia: 1989-2004. Equine Vet J 38: 312-318.

4. Estberg L, Stover SM, Gardner IA, Johnson BJ, Jack RA, Case JT, Ardans B, Read DH, Anderson ML, Barr BC, Daft BM, Kinde H, Moore J, Stoltz J, Woods L. 1998. Relationship between race start characteristics and risk of catastrophic injury in thoroughbreds: 78 cases (1992). J Am Vet Med Assoc 15: 544-549.

5. Melling M, Alder M. 1998. Equine practice 3. Philadelphia, USA: WB Saunders. 233 p.
6. Nixon, A. 1996. Equine fracture repair. New York: WB Saunders. 384 p.

7. Peloso J, Mundy G, Honnas C. 1994. Epidemiologic study musculoskeletal injuries in racing Thoroughbred horses in Kentucky. $42^{\text {th }}$ Annual Convention of the American Association of Equine Practitioners. Denver, Colorado.

8. Sucre L, Hernández, N, Hecker-Torres S. 1999. Efecto del entrenamiento sobre actividades enzimáticas y composición fibrilar en el m.g. medius de caballos pura sangre venezolanos. Rev Cien FCV-LUZ 9: 489-501.

9. Riggs CM. 2002. Fractures - A preventable hazard of racing Thoroughbreds? Vet J 1: 5-6.

10. Rose R, Hodgson D. 1995. Manual clínico de equinos. México DF: Interamericana McGraw-Hill. 205 p.

11. Williams R, Harkins L, Hammond J. 2001. Racehorse injuries, clinical problems and fatalities recorded on British racecourses from flat racing and National Hunt racing during 1996, 1997 and 1998. Equine Vet J 33: 478-486. 